\title{
Motion Control of a Semi-Mobile Haptic Interface for Extended Range Telepresence
}

\author{
Antonia Pérez Arias and Uwe D. Hanebeck
}

\begin{abstract}
This paper presents the control concept of a semimobile haptic interface for extended range telepresence that enables the user to explore spatially unrestricted target environments even from a small user environment. The semi-mobile haptic interface consists of a haptic manipulator mounted on a large grounded Cartesian robot, the prepositioning unit. The prepositioning unit is controlled in such a way that the haptic manipulator is kept off its workspace limits. At the same time, the control algorithm allows the optimal utilization of the available space in the user environment and guarantees the safety of the user. The proposed control method is based on the position and velocity of the end-effector and also takes the position of the user into account. Moreover, it is robust against noisy measurements of the user position or outliers due, for example, to occlusions in the tracking system. Experimental results show the suitability of the proposed control to provide haptic interaction in extended range telepresence.
\end{abstract}

\section{INTRODUCTION}

\section{A. Motivation}

Telepresence systems provide a human operator with the feeling of actual presence in a remote environment, the target environment. The feeling of presence is achieved by visual and acoustic sensory information recorded from the target environment and presented to the user on an immersive display. In order to use the sense of motion as well, which is especially important for human navigation and path finding [1], the user's motion is tracked and transferred to the proxy in the target environment. As a result, in extended range telepresence the operator can additionally use the proprioception, i.e., the sense of motion, to navigate the teleoperator by natural walking, instead of using devices like joysticks, pedals, or steering wheels.

Without further processing of the motion information, the motion of the operator is restricted to the size of the user environment, which is limited, for example, by the range of the tracking system or the available space. Motion Compression [2] solves this problem by mapping the desired path in the target environment (target path) to a feasible path in the user environment (user path) while minimizing proprioceptive and visual inconsistencies. The resulting user path conserves the length and turning angles of the target path while there is a minimum difference in curvature. Finally, the user is guided on the user path, while he has the impression of walking on the original target path. As a result, Motion Compression provides a nonlinear mapping

Antonia Pérez Arias and Uwe D. Hanebeck are with the Intelligent Sensor-Actuator-Systems Laboratory (ISAS), Institute for Anthropomatics, Karlsruhe Institute of Technology (KIT), Karlsruhe, Germany, antonia.perezakit.edu, uwe.hanebeck@ieee.org

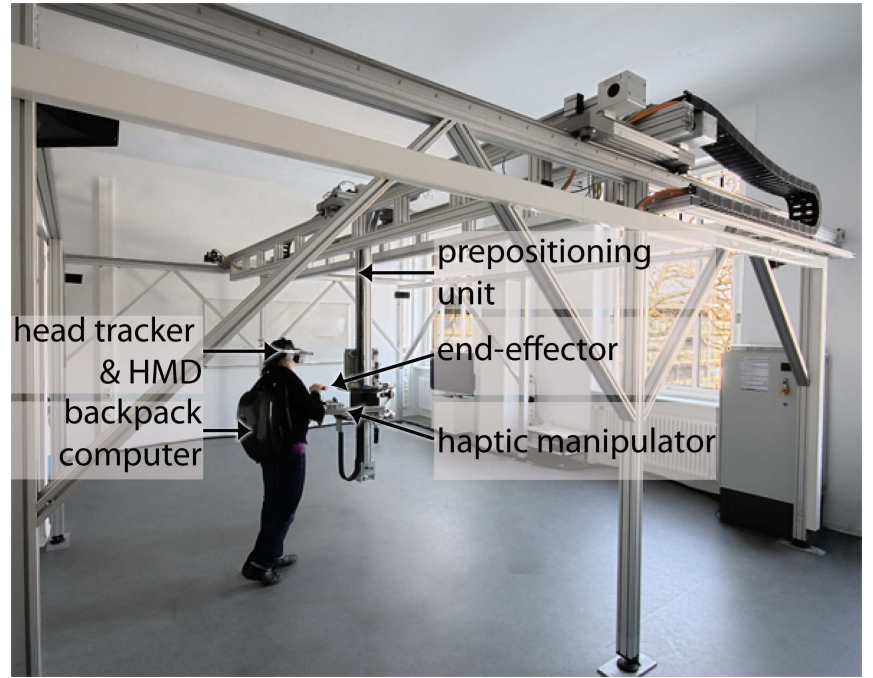

Fig. 1. Semi-mobile haptic interface and operator in the user environment.

between the user's path in the user environment and the path in the target environment. This transformation can also be used to map the user's hand position, or to transform force vectors recorded in the target environment back into the user environment.

Haptic information from the target environment is indispensable, so that the user can perceive objects and obstacles in the target environment more realistically. For this purpose, a semi-mobile haptic interface that allows for simultaneous haptic interaction and wide-area motion was developed [3]. Fig. 1 shows the user interface in our extended range telepresence system.

The semi-mobile haptic interface consists of a haptic manipulator and a prepositioning unit (PPU), which is a grounded robotic system. The haptic manipulator displays defined forces to the human operator through the endeffector. The PPU moves the haptic manipulator along with the user. The prepositioning algorithm has to control the motion of the manipulator in such a way that ...

1) ... the manipulator does not reach the limits of its workspace. Since the user moves his/her arms very fast, the prepositioning algorithm has to keep the haptic manipulator away from its singularities.

2) ... the manipulator does not restrict the motion of the human operator. In particular, the collision-free movement of the manipulator must be guaranteed since the user and the manipulator share the workspace and the user is not aware of the motion of the PPU. 
3) ... the user is able to make use of the whole available space in the user environment. A reduction of the reachable space would cause an increment of the curvature of the user path and this would affect the immersion of the user in the target environment.

\section{B. State of the Art}

Force reflecting telepresence systems usually assume an immobile user and a restricted workspace. For example, industrial robots [4] have often been used as haptic interfaces due to their accuracy and relative high force capability. A novel grounded hyper-redundant haptic interface is presented in [5]. However, the limited workspace of these interfaces makes them unfeasible for extended range telepresence.

Portable haptic interfaces like exoskeletons [6], [7] solve the problem of wide area motion, since the interface is carried along by the user. However, the haptic rendering with these devices is of significantly lower quality than with grounded displays [8] and depends on the localization of the user. The maximum force that can be displayed by an exoskeleton is limited by the weight of the system, which must be carried by the user, and because the displayed force is transmitted to the body of the human operator.

Mobile haptic interfaces [9]-[11] are really suitable for haptic interaction during wide area motion. They are usually haptic devices mounted on a mobile platform. The quality of the haptic rendering of such interfaces strongly depends on the quality of the localization and the position control of the platform. Furthermore, the transparency of the forcecontrolled subsystem can be affected by the compliance of the wheels of the motion subsystem [9].

Mobile haptic interfaces also require an adequate positioning of the haptic display in order to allow for extended range telepresence. In [9], the position of the mobile base is calculated by maximizing a manipulability measure. [12] and [13] include the human arm workspace in the position optimization strategy. This strategy offers advantages when the operator performs fast motions using the full workspace of his arm. However, if the operator performs fast motions using his legs, the performance can deteriorate as the haptic interface is operated closer to its workspace limits. In [10], the motion planning algorithm uses only information about the end-effector position, since their mobile haptic interface is equipped with a visual screen and does not use an external tracker. This approach, although efficient, may fail to prevent a collision between a user and the mobile haptic interface.

In previous work [3], we developed a prepositioning algorithm that takes the user position into account. The position of the PPU is chosen in such a way that the distance from the user is maximized. However, this solution consumes too much space in the user environment as the user must walk at this maximum distance from the limits of the PPU. For this reason, it is necessary to find an adequate prepositioning algorithm that maximizes the space in which the user can move freely.

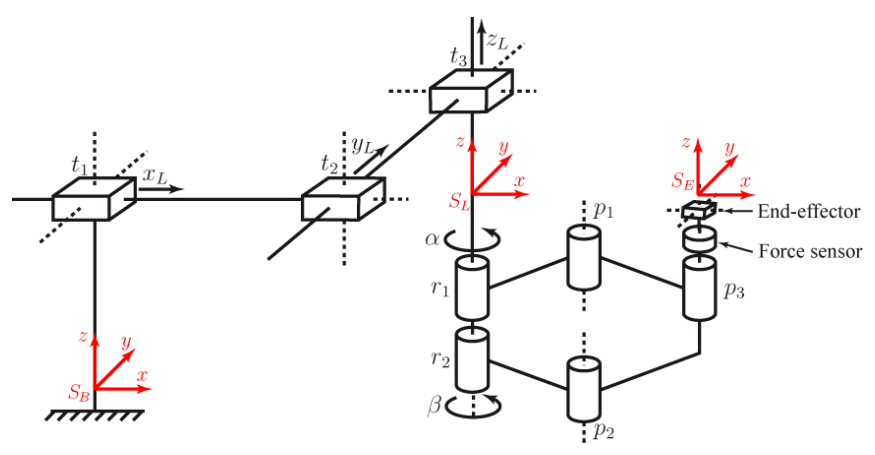

Fig. 2. Kinematic model of a semi-mobile haptic interface. Joints $t_{1}, t_{2}$, $t_{3}$ position the haptic manipulator at $S_{L}$, joints $r_{1}$ and $r_{2}$ determine the the end-effector pose at $S_{E}$. This haptic interface is redundant in the planar degrees of freedom.

\section{Contribution}

None of the previous approaches takes the direction of motion of the user into account. By taking not only the position of the human operator but also his direction of motion into account, the performance of the prepositioning can be significantly improved.

The presented algorithm allows for an optimal prepositioning of the haptic manipulator that enables the user to walk and feel forces even close to the spatial limits of the user environment. The prepositioning algorithm also guarantees the collision-free motion of the manipulator while sharing its workspace with the operator.

A further benefit of our approach is the use of a virtual head position generated from the direction of motion of the hand. The use of this virtual head position allows the prepositioning of the manipulator even without measurements of the real human position and makes the algorithm robust in case of noisy or out-of-range measurements of the user position.

The work is structured as follows. The following Section presents the concept of a semi-mobile haptic interface, as it determines the requirements on the prepositioning algorithm. In Section III, the overall control structure is presented. Experimental results are shown in Section IV. Finally, a summary and an outlook can be found in Section V.

\section{Semi-Mobile Haptic InTERface}

Semi-mobile haptic interfaces combine the benefits of mobile haptic interfaces and grounded haptic interfaces. Like mobile haptic interfaces, they permit wide-area motion together with haptic interaction inside the user environment. They also provide high force capability and accurate haptic rendering like grounded haptic interfaces. Moreover, in combination with Motion Compression they permit the exploration of arbitrarily large target environments from the limited user environment.

A semi-mobile haptic interface consists of two subsystems: a prepositioning unit (PPU) and a haptic manipulator. The PPU controls the position of the manipulator's basis in the user environment, so that the end-effector remains within the workspace of the manipulator. The acceleration of the human hand is typically much higher than the acceleration 
of the PPU. Therefore, in order to allow for natural hand motion, a fast and lightweight manipulator is attached to the PPU. An exemplary realization of a semi-mobile haptic interface is shown in Fig. 2, which is built out of a Cartesian PPU and a parallel planar manipulator.

The main difference with respect to mobile haptic interfaces is that the PPU is a grounded robotic system that covers the whole user environment. This construction has the advantages of a high force capability and an accurate localization of the manipulator's basis, which can be directly determined through the position of the joint encoders. By choosing Cartesian kinematics for the PPU, a high rigidity and a simple position control are achieved.

The control of a semi-mobile haptic interface is based on the decoupling of force control of the haptic manipulator and motion control of the PPU. This separation is possible due to the redundant degrees of freedom that are assumed to be present in both manipulator and PPU. However, this redundancy has to be resolved in order to control the motion of the PPU, as we will explain in the next section.

\section{Control Design}

\section{A. Overall Control Structure}

The overall control structure of the semi-mobile haptic interface is depicted in Fig. 3. The haptic display is modelled as an admittance that transforms the external forces (the reference force $\underline{F}_{r e f}$ from the target environment and the force applied by the user $\underline{F}_{H}$ ) into the desired motion of the end-effector.

In contrast to impedance control, which is frequently used for light and highly backdrivable devices, admittance control is especially well suited for the control of haptic interfaces with high dynamics and nonlinearities [14], which is the case of the semi-mobile haptic interface. The admittance control requires the measurement of the force applied by the user at the end-effector in order to compensate the natural device dynamics. Moreover, since the motion of the PPU is coupled with the end-effector by means of the force sensor, the user does not perceive any motion when the PPU moves.

The admittance model shapes the desired dynamics of the device and describes the desired motion of the end-effector under the influence of the external forces as follows

$$
\underline{F}_{r e f}-\underline{F}_{H}=\boldsymbol{M}_{\boldsymbol{m}} \underline{\ddot{\ddot{x}}}_{E, r e f}+\boldsymbol{D}_{\boldsymbol{m}} \underline{\dot{x}}_{E, r e f}+\boldsymbol{K}_{\boldsymbol{m}} \underline{x}_{E, r e f},
$$

where $M_{m}$ is the mass matrix of the displayed virtual object and $\boldsymbol{D}_{m}$, and $\boldsymbol{K}_{m}$ are matrices that represent the viscous damping and the stiffness of the environment, respectively. The reference position of the end-effector $\underline{x}_{E, r e f}$ is the input of the motion controller, in our case, a computed torque position controller.

To allow for wide-area haptic interaction, the position of the end-effector $\underline{x}_{E}$ is transformed with Motion Compression and sent to the target environment. At the same time, the contact forces from the target environment are transformed with the inverse Motion Compression transformation and presented to the operator as $\underline{F}_{r e f}$. In order to compensate for possible position drifts between the transformed endeffector position and the current position of the proxy in the target environment, this admittance control can be extended by adding a feedforward term (proportional to the position drift) to the motion controller. In Fig. 3, this term is omitted for the sake of clarity.

The position of the controlled manipulator is obtained from the position of the PPU $\underline{x}_{L}$ and the position of the end-effector w.r.t. the PPU $\underline{x}_{S}$, which depends only on the actual manipulator configuration, as

$$
\underline{x}_{E}=\underline{x}_{L}+\underline{x}_{S} .
$$

The goal of the motion control of the PPU is to place the workspace of the manipulator at the user's disposal at any time. Given the actual position of the end-effector and assuming redundancy of the system in the planar degrees of freedom, the PPU is controlled within the null-space in order to maximize the manipulability of the haptic device. The optimal position of the PPU $\underline{x}_{L, r e f}$, which is the input of its position controller, is obtained as

$$
\underline{x}_{L, r e f}=\underline{x}_{E}-\underline{x}_{S, \text { opt }},
$$

where $\underline{x}_{S, \text { opt }}$ is the optimal configuration of the manipulator regarding a certain manipulability measure.

By doing so, the PPU follows the end-effector and tries to maintain the optimal configuration of the manipulator. Since the velocity bandwidth of the haptic manipulator is higher than the bandwidth of the PPU, fast motions of the end-effector are handled by the haptic manipulator, while slow motions are handled by the PPU by keeping the manipulator near its optimum manipulability. This strategy is in accordance with the fact that the user's walking motion is slower than the user's arm motions.

\section{B. Maximizing Manipulability}

There are different manipulability measures that describe the output capability of manipulators depending on the actual joint configuration. The most common one is the velocity manipulability (also known as Yoshikawa's measure) that describes the ability of the manipulator to generate velocity and degenerates close to singular configurations [15]. The velocity manipulability $w$ of the haptic display for a certain configuration $\gamma$ represents the volume of the manipulability ellipsoid and by maximizing this value, the distance to all singularities is maximized.

We are thus interested in the optimal configuration $\underline{\gamma}_{o p t}$ that maximizes the manipulability $w(\underline{\gamma})$ so that

$$
\underline{\gamma}_{o p t}=\arg \max _{\underline{\gamma}}\{w(\underline{\gamma})\} .
$$

The manipulability of our haptic display is only affected by the radial distance of the end-effector and is constant on circles around the PPU of radius $r_{o p t}$. Thus, given an endeffector position $\underline{x}_{E}$, the optimal reference position $\underline{x}_{L, \text { ref }}$ is located at a distance $r_{\text {opt }}$ from the end-effector. Since the position of the PPU is still undefined, the position of the user shall be taken into account in order to determine the 


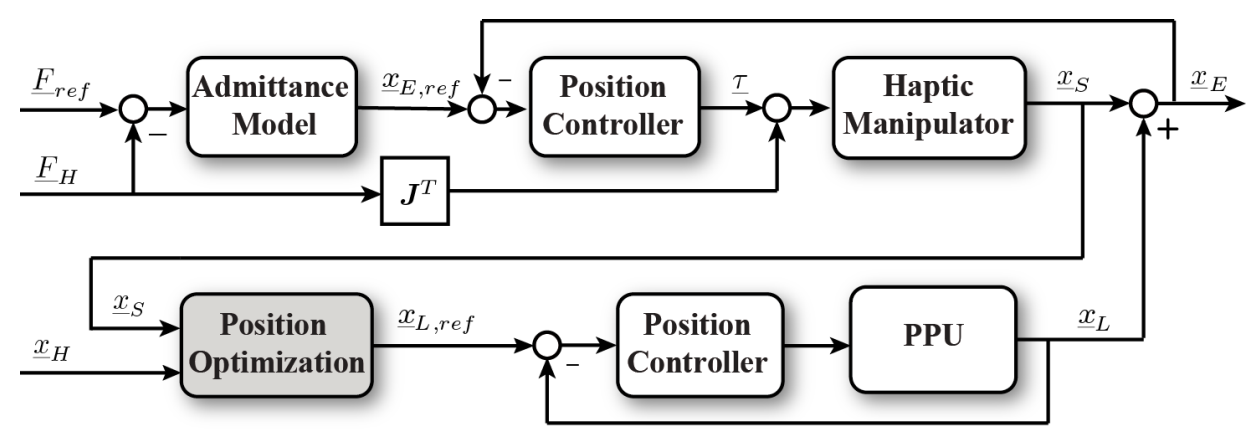

Fig. 3. Overall control structure of the haptic interface.

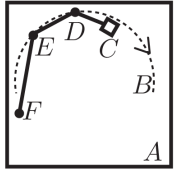

(a)

A... user environment

$B$... user path

$C$... desired PPU position

$D$... user's hand position

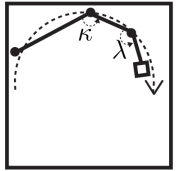

(b)

E... user position

$F$... tail position

$\kappa \ldots$ head angle

$\lambda \ldots$ hand angle
Fig. 4. Positioning algorithm for one typical motion sequence.

optimal position of the PPU that does not interfere with the user motion.

\section{Including Direction of Motion}

The position of the PPU should be chosen in such a way that the PPU does not interfere with the motion of the user. In addition to this, the prepositioning algorithm has to permit the maximum utilization of the available space in the user environment. For this purpose, we assume that the user walks forward, which is a realistic assumption when Motion Compression is used to walk in arbitrarily large target environments. While following a piecewise straight path in the arbitrarily large target environment, the user walks tangential to a curved user path that fits into the user environment. The PPU has to be prepositioned in such a way that the user is able to walk and move the end-effector on this path without reaching the singularities of the haptic manipulator.

The key idea of this optimal prepositioning lies in estimating the instantaneous curvature of the user path and calculating the reference position of the PPU that adjusts itself to the curvature of the path. We could use the instantaneous transformation provided by Motion Compression in order to transform the position of the PPU to a feasible position on the user path. However, this transformation can change rapidly, since it also depends on the human view direction, and does not account for user's arm motions. Therefore, we use instead a rough estimation of the path curvature based on current motion data of the user in the user environment.

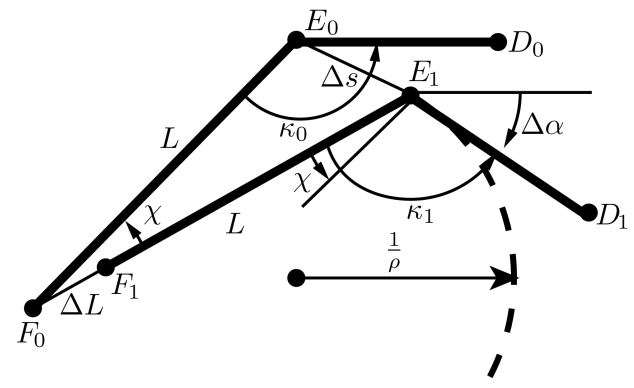

Fig. 5. Calculation of new hand angle $\kappa_{1}$ using the instantaneous path curvature $\frac{1}{\rho}$ and the increment on the position of the user $\Delta s$.

Our approach makes use of a virtual object that provides for a slow change of the orientation of the PPU. This virtual object, which will be called tail, is attached to the user by a virtual rope, so that motions of the user and the user's hand lead to displacements of the virtual rope and the tail position.

Fig. 4 illustrates the prepositioning algorithm. If the user walks on a straight path forwards, the rope will be aligned with the user and in this case $\kappa=\pi$. However, when the path is curved, $\kappa$ decreases or increases depending if the user walks on a right curved path (Fig. 4(a), Fig. 4(b)) or on a left curved path (Fig. 4(c)), respectively. By positioning the PPU in such a way that the angle $\lambda$ at the hand position is $\lambda=\kappa$, the haptic display will always be positioned inside the curved path.

Fig. 5 shows in more detail how the angle $\kappa$ is calculated. The initial head angle $\kappa_{0}$ is defined by $F_{0}, E_{0}$, and $D_{0}$, the initial positions of the tail, the user, and the hand of the user, respectively. Assuming that the user walks a distance $\Delta s$ on the user path, the head angle at the next step $\kappa_{1}$ can be calculated as

$$
\kappa_{1}=\kappa_{0}+\Delta \alpha+\chi
$$

where $\Delta \alpha$ accounts for the change in the orientation of the user due to the instantaneous path curvature $\frac{1}{\rho}$ and $\chi$ accounts for the motion of the tail using a virtual rope of length $L$. Actually, the path curvature $\frac{1}{\rho}$ can be expressed as

$$
\frac{1}{\rho}=\frac{\Delta \alpha}{\Delta s}
$$


A straightforward calculation of $\chi$ yields

$$
\chi=\arccos \left(\frac{L-\Delta s \cos \left(\kappa_{0}+\Delta \alpha\right)}{L+\Delta L}\right) .
$$

Furthermore, the length of the rope $L$ can be adjusted to increase or decrease the influence of the instantaneous path curvature: increasing the length of the rope leads to a decrease of $\chi$ so that the limit for $\kappa$ when $L$ approaches $\infty$ yields

$$
\lim _{L \rightarrow \infty} \kappa_{1}=\kappa_{0}+\Delta \alpha
$$

where only the curvature is considered. As opposed to this, decreasing the length $L$ diminishes the effect of the path curvature and the limit calculation yields

$$
\lim _{L \rightarrow 0} \kappa_{1}=\pi .
$$

In this case, the head angle is independent of the curvature. Please note that the maximum distance approach proposed in [3], where the desired position of the PPU is calculated by maximizing the distance between the operator and the haptic interface, is a special case of this approach in which the length of the rope is $L=0$.

By combining both the motion of the user and the motion of the user's hand, this method succeeds in adjusting the motion of the PPU to the current curvature of the user path as well as in following the fast motions of the user's hand around his body, which obviously lead, as in the case of the maximum distance approach, to fast rotations of the PPU around the user.

\section{Virtual Head Position}

If we assume that the user walks forward while keeping his hand in front of the body, a virtual head position attached to the user's hand by a virtual rope can be calculated in a similar fashion to the tail position. The reference position of the PPU can then be calculated without measurements of the user's head position by substituting the user's head position by the virtual head position.

In order to account for the motion of the user's hand toward the virtual head that can be produced because the user walks backwards or moves the arm back toward his body, a safety region around the virtual head position is defined. If the end-effector enters this region, the virtual head and the tail will follow the motion of the end-effector backwards as if they were rigidly connected. This region avoids that the virtual head comes too close to the user's hand, in which case the haptic display would suddenly change its orientation with respect to the user. In order to keep a minimum distance between the haptic display and the human operator the angle $\kappa$ is bounded.

The assumption of the user walking forward while moving his hand in front of the body is only plausible when the user walks in free space. However, if measurements of the position of the user are available, the virtual position can easily be merged with the actual position of the user to achieve a feasible prepositioning even when the assumption of the user walking with his hand in front of the body is not correct. The use of the virtual head position is also beneficial when dealing with noisy or inaccurate measurements of the position of the operator.

\section{EXPERIMENTAL RESULTS}

In order to illustrate the capabilities of the novel prepositioning approach as well as the benefits resulting from considering a virtual head position, several test runs under real-world conditions were conducted. The haptic interface used to perform the experiments is an implementation of the concept of a semi-mobile haptic interface introduced in section II and will be described below.

\section{A. Experimental Setup}

The semi-mobile haptic interface (Fig. 1) consists of two subsystems. The linear PPU is realized as a grounded portal carrier system of approximately $4 \times 4 \mathrm{~m}^{2}$. Each axis of the PPU consists of two parallel rails driven by synchronous AC motors. A magnetic measuring system mounted on the rails provides the position of the PPU with a resolution of $0.1 \mathrm{~mm}$.

The manipulator arm is realized as a parallel Selective Compliance Assembly Robot Arm (SCARA) with four links: two internal links of length of $l_{1}=0.285 \mathrm{~m}$ and two external links with $l_{2}=0.708 \mathrm{~m}$. The active rotational joints driven by two $150 \mathrm{~W}$ DC motors are integrated into the base, so that the mass of all moving parts is about $12 \mathrm{~kg}$. Since circular guides are used to drive the links, the workspace of the arm is a hollow cylinder with external radius $r_{e}=l_{1}+l_{2}=0.993 \mathrm{~m}$ and internal radius $r_{i}=l_{1}-l_{2}=0.423 \mathrm{~m}$. The manipulator arm is able to display forces up to $150 \mathrm{~N}$. The maximum velocity and acceleration of the end-effector are $0.98 \mathrm{~m} / \mathrm{s}$ and $12.5 \mathrm{~m} / \mathrm{s}^{2}$, respectively, and the position resolution at the end-effector is $0.1 \mathrm{~mm}$ as well. The force bandwidth of the haptic manipulator is about $8 \mathrm{~Hz}$.

\section{B. Scenario}

The user can walk on a surface of $4 \times 4 \mathrm{~m}^{2}$ in the user environment. However, for safety reasons and to avoid a possible damage of the haptic interface, the boundaries of the Cartesian workspace lie inside the user environment, so that the Cartesian workspace of the haptic interface is only $2.5 \times 3.5 \mathrm{~m}^{2}$ large. The PPU cannot move beyond these boundaries and is controlled to keep a distance $r_{\text {opt }}=0.805$ from the end-effector. The limits of the workspace of the haptic manipulator are situated at distances $r_{e}=0.993 \mathrm{~m}$ and $r_{i}=0.423 \mathrm{~m}$ from the PPU.

The length of the virtual rope between the virtual head position and the hand position is $0.5 \mathrm{~m}$. When the distance between hand and virtual head is smaller than $0.3 \mathrm{~m}$, then virtual head and tail move parallel to the hand position. The length of the virtual rope between the tail and the virtual head is $5 \mathrm{~m}$. $\kappa$ was bounded so that $2 \pi / 3<\kappa<4 \pi / 3$. The desired position of the haptic manipulator was controlled by using a high gain linear PD controller.

Two scenarios were chosen for evaluating the proposed algorithm. For the first scenario, a typical task of walking 
on a long $(20 \mathrm{~m})$ straight target path and consequently on a curved path in the user environment was chosen. In the second scenario, the user interacts with two virtual walls following their contours with his hand. For benchmarking the results of these experiments, we compared our present approach to the previous maximum distance method [3].

\section{Results}

With the maximum distance method, the PPU quickly reaches the Cartesian limits of the haptic interface in both scenarios. When the user walks further forward, the endeffector reaches also the internal singularity at $r_{i}=0.423 \mathrm{~m}$. At this configuration, the end-effector suddenly comes to a stop, which is perceived as disturbing by the human operator.

Fig. 6 shows the trajectories of end-effector (hand position) and PPU for the first scenario and clearly shows that the propositioning employing our novel approach manages to keep the end-effector inside the workspace of the manipulator, even without taking the actual position of the operator into account.

Fig. 7 shows the trajectories of user (head position), endeffector (hand position), and PPU for the second scenario. Please note that in Fig. 7(b.1) and Fig. 7(c.1), the PPU always precedes the user and thus, they never collide. The apparent intersections of the trajectories of user and PPU and/or endeffector are due to the fact the temporal coordinate is missing in the plots, i.e., PPU, user, and/or end-effector reach the same positions but in different time instants. These results indicate that the proposed approach is also feasible when the user does not walk with his hand in front of his body and interacts with the virtual environment instead.

\section{Conclusions}

In this work, we proposed a motion control method for a semi-mobile haptic interface that provides both a large workspace and a high force capability. In conjunction with Motion Compression, the presented control method allows for haptic exploration of spatially unrestricted target environments from a limited user environment.

The control of the semi-mobile haptic interface is based on the decoupling of force control and wide-area motion control of the haptic device. The presented position control not only takes the position of the human operator into account, but also his direction of motion. It allows for an optimal positioning of the haptic manipulator away from its workspace limits, guarantees the safety of the operator, and permits the display of forces even close to the spatial limits of the user environment.

A further benefit of our approach is the use of a virtual head position generated from the direction of motion of the user's hand. The use of this virtual head position permits the motion control of the interface even without measurements of the user position and makes the algorithm robust against noisy or out-of-range measurements of the user position as we demonstrated in real-world experiments. The proposed algorithm can also be used for mobile haptic interfaces, with or without external user tracker.

Current work is concerned with quantifying the performance of the prepositioning method depending on the feasible combined motions of user and user's hand in order to adapt the control parameters online.

\section{REFERENCES}

[1] R. P. Darken, T. Allard, and L. B. Achille, "Spatial Orientation and Wayfinding in Large-Scale Virtual Spaces: An Introduction," Presence, vol. 7, no. 2, pp. 101-107, Apr. 1998.

[2] N. Nitzsche, U. D. Hanebeck, and G. Schmidt, "Motion Compression for Telepresent Walking in Large Target Environments," Presence: Teleoperators and Virtual Environments, vol. 13, no. 1, pp. 44-60, Feb. 2004.

[3] A. Pérez Arias and U. D. Hanebeck, "A Novel Haptic Interface for Extended Range Telepresence: Control and Evaluation," in Proceedings of the 6th International Conference on Informatics in Control, Automation and Robotics (ICINCO 2009), Milan, Italy, July 2009, pp. 222-227.

[4] J. Hoogen and G. Schmidt, "Experimental Results in Control of an Industrial Robot Used as a Haptic Interface," in Proceedings of the IFAC Telematics Applications in Automation and Robotics, 2001.

[5] M. Ueberle, N. Mock, and M. Buss, "Towards a Hyper-Redundant Haptic Display," in Proceedings of the International Workshop on High-Fidelity Telepresence and Teleaction, 2003.

[6] N. Tsagarakis, D. G. Caldwell, and G. A. Medrano-Cerda, "A 7 DOF Pneumatic Muscle Actuator (pMA) Powered Exoskeleton," in Proceedings of the 8th IEEE Int. Workshop Robot and Human Interaction, 1999, pp. 327-333.

[7] A. Frisoli, F. Rocchi, S. Marcheschi, A. Dettori, F. Salsedo, and M. Bergamasco, "A New Force-Feedback Arm Exoskeleton for Haptic Interaction in Virtual Environments," in Proceedings of the first Joint Eurohaptics Conf. and Symp. Haptic Interfaces for Virtual Environment and Teleoperator Systems, 2005, pp. 195-201.

[8] C. Richard and M. R. Cutkosky, "Contact Force Perception with an Ungrounded Haptic Interface," in Proceedings of ASME IMECE 6th Annual Symposium on Haptic Interfaces, 1997.

[9] N. Nitzsche, U. D. Hanebeck, and G. Schmidt, "Design Issues of Mobile Haptic Interfaces," Journal of Robotic Systems, vol. 20:9, pp. 549-556, 2003.

[10] A. Formaglio, D. Prattichizzo, F. Barbagli, and A. Giannitrapani, "Dynamic Performance of Mobile Haptic Interfaces," IEEE Transactions on Robotics, vol. 24, no. 3, pp. 559-575, 2008.

[11] A. Peer, Y. Komoguchi, and M. Buss, "Towards a Mobile Haptic Interface for Bimanual Manipulations," in Proceedings of the IEEE/RSJ International Conference on Intelligent Robots and Systems, 2007, pp. 384-391.

[12] U. Unterhinninghofen, T. Schauß, and M. Buss, "Control of a Mobile Haptic Interface," in Proceedings of the IEEE International Conference on Robotics and Automation, 2008, pp. 2085-2090.

[13] I. Lee, I. Hwang, K.-L. Han, O. K. Choi, S. Choi, and J. S. Lee, "System Improvements in Mobile Haptic Interface," in Proceedings of the third Joint Eurohaptics Conference and Symposium on Haptic Interfaces for Virtual Environment and Teleoperator Systems, 2009, pp. 109-114.

[14] M. Ueberle and M. Buss, "Control of Kinesthetic Haptic Interfaces," in Proceedings of the IEEE/RSJ Intl. Conference on Intelligent Robots and Systems, Workshop on Touch and Haptics, 2004.

[15] T. Yoshikawa, "Manipulability and Redundancy Control of Robotic Mechanisms," in Proceedings of IEEE Intl. Conference on Robotics and Automation, 1985, pp. 1004-1009. 


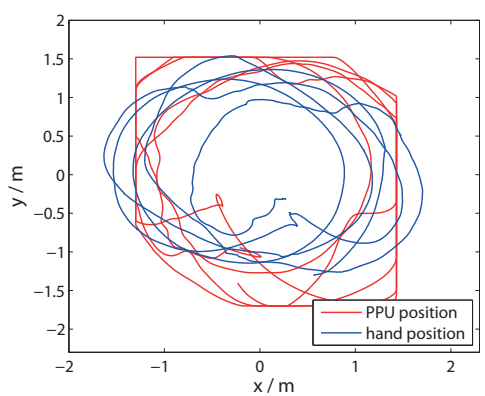

(a.1)

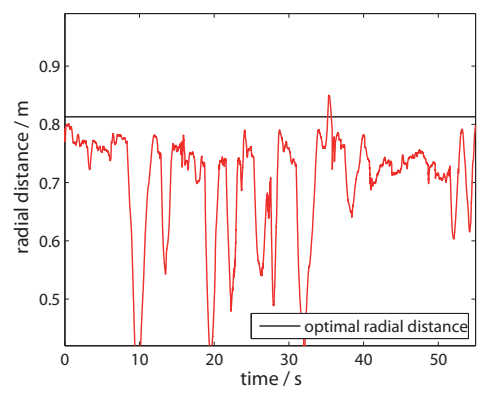

(a.2)

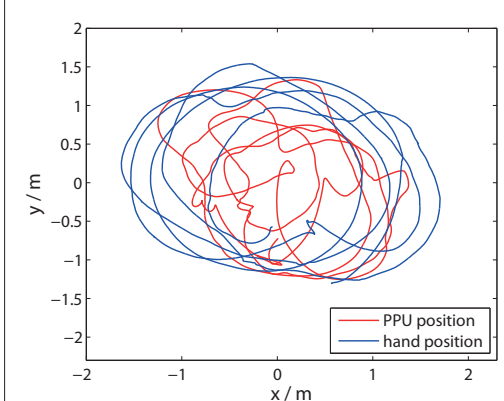

(b.1)

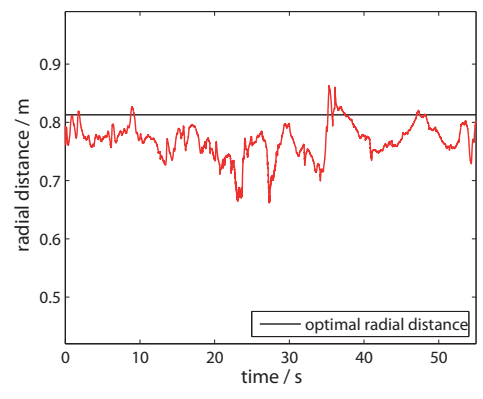

(b.2)

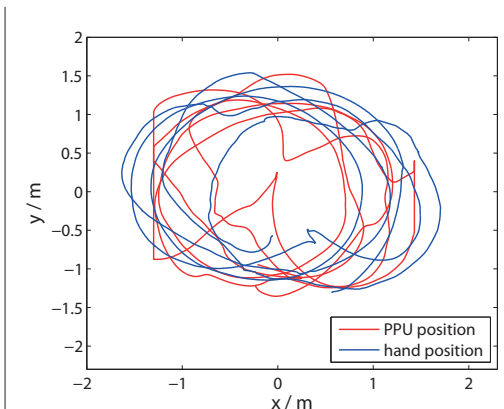

(c.1)

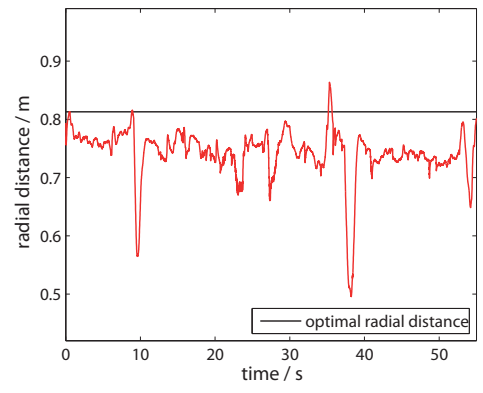

(c.2)

Fig. 6. Experimental runs: (a.1) and (a.2) were realized with the maximum distance method, (b.1), (b.2) with the proposed approach. (c.1), (c.2) were performed without using measurements of the user position. Instead of that, the virtual head position was inferred using the motion of the end-effector. The figures on the first row show the trajectories of the end-effector and the PPU in the user environment, whereas the figures on the second row show the distance between the end-effector and the PPU during the test run. The limits of these plots on the vertical axis correspond with the limits of the workspace of the haptic display.

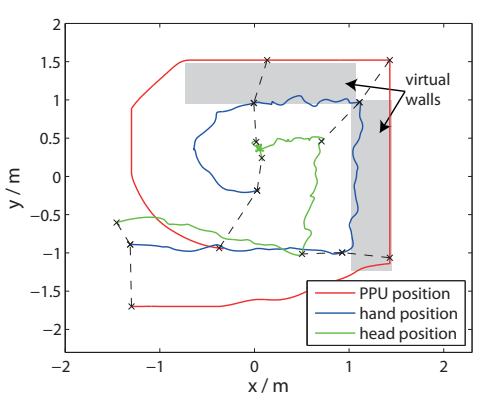

(a.1)

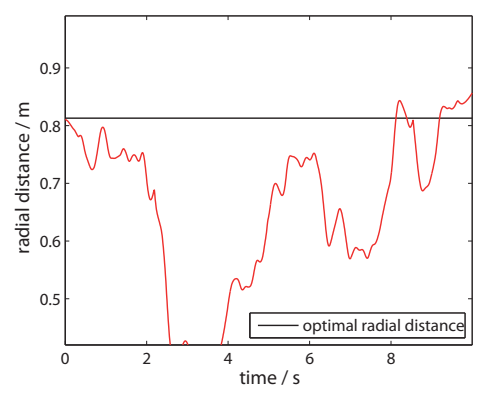

(a.2)

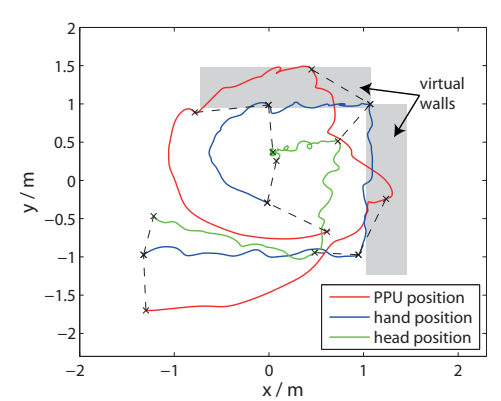

(b.1)

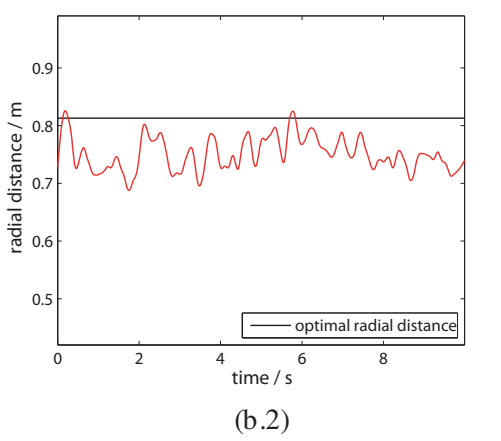

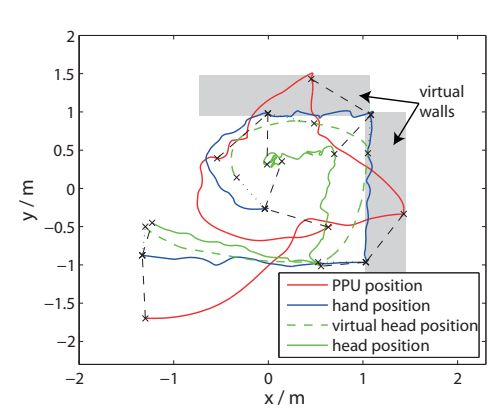

(c.1)

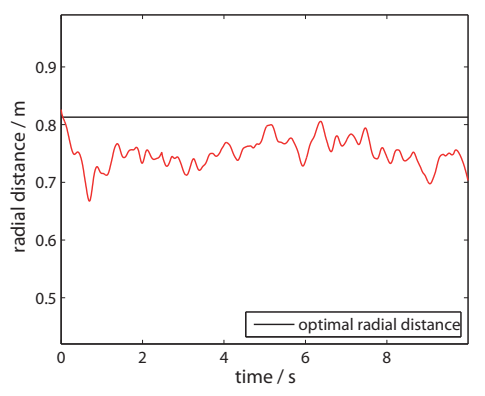

(c.2)

Fig. 7. Experimental runs: (a.1) and (a.2) were realized with the maximum distance method, (b.1), (b.2) with the proposed approach, and (c.1), (c.2) using the virtual head position. 